\title{
Generalized Derivations on Power Values of Lie Ideals in Prime and Semiprime Rings
}

\author{
Vincenzo De Filippis, ${ }^{1}$ Nadeem UR Rehman, ${ }^{2}$ and Abu Zaid Ansari ${ }^{3}$ \\ ${ }^{1}$ Department of Mathematics and Computer Science, University of Messina, Viale Ferdinando Stagno d'Alcontres 31, \\ 98166 Messina, Italy \\ ${ }^{2}$ Department of Mathematics, Aligarh Muslim University, Aligarh 202002, India \\ ${ }^{3}$ Faculty of Science, Islamic University in Madinah, P.O. Box 170, Madinah 42351, Saudi Arabia
}

Correspondence should be addressed to Vincenzo De Filippis; defilippis@unime.it

Received 3 January 2014; Accepted 12 May 2014; Published 3 June 2014

Academic Editor: Kaiming Zhao

Copyright (C) 2014 Vincenzo De Filippis et al. This is an open access article distributed under the Creative Commons Attribution License, which permits unrestricted use, distribution, and reproduction in any medium, provided the original work is properly cited.

Let $R$ be a 2-torsion free ring and let $L$ be a noncentral Lie ideal of $R$, and let $F: R \rightarrow R$ and $G: R \rightarrow R$ be two generalized derivations of $R$. We will analyse the structure of $R$ in the following cases: (a) $R$ is prime and $F\left(u^{m}\right)=G\left(u^{n}\right)$ for all $u \in L$ and fixed positive integers $m \neq n$; (b) $R$ is prime and $F\left(\left(u^{p} v^{q}\right)^{m}\right)=G\left(\left(v^{r} u^{s}\right)^{n}\right)$ for all $u, v \in L$ and fixed integers $m, n, p, q, r, s \geq 1$; (c) $R$ is semiprime and $F\left((u v)^{n}\right)=G\left((v u)^{n}\right)$ for all $u, v \in[R, R]$ and fixed integer $n \geq 1$; and (d) $R$ is semiprime and $F\left((u v)^{n}\right)=G\left((v u)^{n}\right)$ for all $u, v \in R$ and fixed integer $n \geq 1$.

\section{Introduction}

Let $R$ be an associative ring with characteristic different from 2, $Z(R)$ its center, $U$ its (right) Utumi quotient ring, and $C$ its extended centroid. The simple commutator $a b-b a$ will be denoted by $[a, b]$. Recall that a derivation $d: R \rightarrow R$ is an additive map satisfying the product rule $d(x y)=d(x) y+$ $x d(y)$ for all $x, y \in R$.

A left multiplier $H$ on a ring $R$ is an additive map satisfying the rule $H(x y)=H(x) y$ for all $x, y \in R$. In case there exists an endomorphism $g$ of $R$ such that $H(x y)=$ $H(x) g(y)$ for all $x, y \in R$, then $H$ is called left $g$-multiplier of $R$.

A generalized derivation $F$ on a ring $R$ is an additive map satisfying $F(x y)=F(x) y+x d(y)$ for all $x, y \in R$ and some derivation $d$ of $R$. A significative example is a map of the form $F(x)=a x+x b$, for some $a, b \in R$; such generalized derivations are called inner. Generalized derivations have been primarily studied on operator algebras. Therefore any investigation from the algebraic point of view might be interesting (see, e.g., [1]). Notice that any derivation is a generalized one and also that the generalized inner derivations include left multipliers and right multipliers. Thus the concept of generalized derivation covers both the concept of derivation and the concept of left (right) multipliers.

Since the sum of two generalized derivations is a generalized derivation, of course every map of the form $F(x)=$ $c x+d(x)$ is a generalized derivation on $R$, where $c$ is a fixed element of $R$ and $d$ is a derivation of $R$.

In [1, Theorem 3] Lee proved that every generalized derivation $F$ on a dense right ideal of $R$ can be uniquely extended to the Utumi quotient ring $U$ of $R$, and thus any generalized derivation of $R$ can be defined on the whole $U$; moreover it is of the form $F(x)=a x+d(x)$ for some $a \in U$ and $d$ is a derivation on $U$ ( $F$ is said to be a generalized derivation associated with derivation $d)$.

Many results in the literature indicate that the global structure of a ring $R$ is often tightly connected to the behaviour of additive mappings defined on $R$.

In [2] Bergen proved that if $g$ is an automorphism of $R$ such that $(g(x)-x)^{m}=0$, for all $x \in R$, where $m \geq 1$ is a fixed integer, then $g=1$. Daif and Bell [3] showed some results which have the same flavour, when the automorphism is replaced by a nonzero derivation $d$. In [3] it is proved that 
if $R$ is a semiprime ring with a nonzero ideal $I$ such that $d([x, y])-[x, y]=0$, or $d([x, y])+[x, y]=0$, for all $x, y \in I$, then $I$ is central. Later Hongan [4] proved that if $R$ is a 2-torsion free semiprime ring and $I$ a nonzero ideal of $R$, then $I$ is central if and only if $d([x, y])-[x, y] \in Z(R)$, or $d([x, y])+[x, y] \in Z(R)$, for all $x, y \in I$. Recently in [5] Ashraf and Ali obtained commutativity theorems for prime rings admitting left multipliers which satisfy similar conditions. More precisely in [5] it is showed that a prime ring $R$ must be commutative if there exist a nonzero ideal $I$ of $R$ and a left multiplier $H$, which is not the identity map on $I$, such that one of the following holds: (i) $H([x, y])=[x, y]$ for all $x, y \in I$ [5, Theorem 2.1]; (ii) $H(x y)-x y \in Z(R)$ for all $x, y \in I$ [5, Theorem 3.1]; and (iii) $H(x y)-y x \in Z(R)$ for all $x, y \in I[5$, Theorem 3.3]. Moreover the same results hold in case $H$ is replaced by a generalized derivation $F$ of $R$.

In a more recent paper [6], Ali and Huang extended the previous cited results in the case $R$ is a semiprime ring, $I$ is a nonzero ideal of $R$, and $H$ is a left $g$-multiplier of $R$. They proved that $I \subseteq Z(R)$ if one of the following holds: (i) $H([x, y])=[x, y]$ for all $x, y \in I[6$, Theorem 2.1]; (ii) $H(x y)=x y$ for all $x, y \in I[6$, Theorem 2.2]; (iii) $H(x y)=y x$ for all $x, y \in I\left[6\right.$, Theorem 2.3]; and (iv) $H\left(x^{2}\right)=x^{2}$, for all $x \in I$ [6, Theorem 2.5]. Moreover, in case $R$ is a prime ring, the same conditions force the commutativity of $R$.

A natural question is to consider additive maps $F, G$ : $R \rightarrow R$ such that $F(f(x, y))=G(g(x, y))$, when $f(x, y)$ and $g(x, y)$ are both either monomials or powers of the commutator $[x, y]$. In this sense, in [7] it is proved that, under appropriate torsion assumptions, a prime $\operatorname{ring} R$ is commutative if it admits a nonzero derivation $d$ satisfying one of the following: (i) $d\left(x^{n} y^{m}\right)=d\left(y^{m} x^{n}\right)$; (ii) $d\left((x y)^{n}\right)=$ $d\left((y x)^{n}\right)$; (iii) $d\left((x y)^{n}\right)=d\left(x^{n} y^{n}\right)$; and (iv) $d\left((x y)^{n}\right)=$ $d\left(y^{n} x^{n}\right)$, for all $x, y \in R$.

So it seems natural to ask about the case when the derivation $d$ is replaced by a generalized derivation. Motivated by the previous cited results, in this paper we will introduce two different generalized derivations acting on $R$ and satisfying some appropriate conditions on some suitable subsets of $R$. We will prove the following.

Theorem 1. Let $R$ be a 2-torsion free prime ring and let $F$ : $R \rightarrow R$ and $G: R \rightarrow R$ be two generalized derivations associated with derivations $d: R \rightarrow R$ and $\delta: R \rightarrow R$, respectively. Suppose that there exist $m, n \geq 1$ integers such that $F\left([x, y]^{m}\right)=G\left([x, y]^{n}\right)$ for all $x, y \in R$. Then either $R$ satisfies the standard identity $s_{4}$ or one of the following holds:

(a) $F=G$ is an inner ordinary derivation of $R$ and for all $x_{1}, x_{2} \in R$ there exists $\alpha \in C$ such that $\left[x_{1}, x_{2}\right]^{m}=$ $\left[x_{1}, x_{2}\right]^{n}+\alpha$;

(b) $F=G$ and $\left[x_{1}, x_{2}\right]^{m}=\left[x_{1}, x_{2}\right]^{n}$, for all $x_{1}, x_{2} \in R$.

Theorem 2. Let $R$ be a 2-torsion free prime ring and let $L$ be a noncentral Lie ideal of $R$, and let $F: R \rightarrow R$ and $G: R \rightarrow R$ be two nonzero generalized derivations associated with derivations $d: R \rightarrow R$ and $\delta: R \rightarrow R$, respectively. Suppose that there exist $m, n, p, q, r, s \geq 1$ integers such that
$F\left(\left(u^{p} v^{q}\right)^{m}\right)=G\left(\left(v^{r} u^{s}\right)^{n}\right)$ for all $u, v \in L$. Then $R$ satisfies the standard identity $s_{4}$.

In the last section we study some commutativity conditions for a semiprime ring with a generalized derivation satisfying suitable algebraic conditions. More precisely, we will prove the following.

Theorem 3. Let $R$ be a 2-torsion free semiprime ring and let $F$ : $R \rightarrow R$ and $G: R \rightarrow R$ be generalized derivations associated, respectively, with derivations $d: R \rightarrow R$ and $\delta: R \rightarrow R$. Set $L=[R, R]$ and $n \geq 1$ such that $F\left((u v)^{n}\right)=G\left((v u)^{n}\right)$ for all $u, v \in L$. Then there exists a central idempotent e of $U$ such that, on the direct sum decomposition $U=e U \oplus(1-e) U$, the generalized derivations $F$ and $G$ vanish identically on $\mathrm{e} U$ and the ring $(1-e) U$ satisfies $s_{4}$.

Theorem 4. Let $R$ be a 2-torsion free semiprime ring and let $F$ : $R \rightarrow R$ and $G: R \rightarrow R$ be generalized derivations associated with derivations $d: R \rightarrow R$ and $\delta: R \rightarrow R$, respectively. Set $n \geq 1$ such that $F\left((u v)^{n}\right)=G\left((v u)^{n}\right)$ for all $u, v \in R$. Then $R$ contains a nonzero central ideal, $d(R) \subseteq Z(R), \delta(R) \subseteq Z(R)$, and there exist $\lambda, \mu \in C$ such that $F(x)=\lambda x+d(x), G(x)=$ $\mu x+\delta(x)$, for all $x \in R$.

Moreover there exists a central idempotente of $U$ such that, on the direct sum decomposition $U=e U \oplus(1-e) U$, the generalized derivations $F$ and $G$ vanish identically on $\mathrm{e} U$ and the ring $(1-e) U$ is commutative.

\section{Action of Generalized Derivations on Prime Rings}

In order to prove the main result in this section, we first fix some intermediate lemmas and theorems. We begin with the following.

Remark 5 (see [8], Lemma 3). Let $R$ be a prime ring with extended centroid $C$ and let $f\left(x_{1}, \ldots, x_{n}\right)$ be any polynomial, not necessarily multilinear. Then the right (left) annihilator of $f\left(x_{1}, \ldots, x_{n}\right)$ is zero, unless when $f\left(x_{1}, \ldots, x_{n}\right)$ is an identity for $R$.

Lemma 6. Let $R$ be a primitive ring which is isomorphic to a dense ring of linear transformations of a vector space $V$ over the field $C$ such that $\operatorname{dim}_{C} V \geq 2$ and let $a, b \in R, m, n \geq 1$, different integers. If for any $x, y \in R,[x, y]^{m} a=[x, y]^{n} b$, then $a=b$ and $\left[x_{1}, x_{2}\right]^{m}=\left[x_{1}, x_{2}\right]^{n}$ for all $x_{1}, x_{2} \in R$, unless when $a=b=0$.

Proof. Let $0 \neq v \in V$. Since $\operatorname{dim}_{C} V \geq 2$, there exists $w \in V$ such that $\{v, w\}$ are linearly $C$-independent. By the density of $R$ there exist $x, y \in R$ such that

$$
v x=w, \quad v y=0, \quad w y=v
$$

so that $v[x, y]=v$ and by the hypothesis

$$
0=v\left([x, y]^{m} a-[x, y]^{n} b\right)=v(a-b)
$$

that is $V(a-b)=(0)$, which implies $a=b$. Hence $\left(\left[x_{1}, x_{2}\right]^{m}-\right.$ $\left.\left[x_{1}, x_{2}\right]^{n}\right) a=0$ for all $x_{1}, x_{2} \in R$ and by Remark 5 we have 
that either $a=b=0$ or $\left[x_{1}, x_{2}\right]^{m}=\left[x_{1}, x_{2}\right]^{n}$ for any $x_{1}$, $x_{2} \in R$.

Lemma 7. Let $R$ be a 2-torsion free prime ring, $J$ a nonzero two-sided ideal of $R$, and $f\left(x_{1}, \ldots, x_{n}\right)$ a noncentral polynomial over $C$, the extended centroid of $R$. If $F: R \rightarrow R$ is a generalized derivation associated with a derivation $d: R \rightarrow R$ such that $F\left(f\left(r_{1}, \ldots, r_{n}\right)\right) \in C$ for all $r_{1}, \ldots, r_{n} \in J$, then $F(x)=0$, for all $x \in R$.

Proof. In light of previous remarks, we have that there exists $a \in U$, the Utumi quotient ring of $R$, such that $F(x)=a x+$ $d(x)$, for all $x \in R$. Thus $J$ satisfies the generalized differential identity

$$
\left[a f\left(x_{1}, \ldots, x_{n}\right)+d\left(f\left(x_{1}, \ldots, x_{n}\right)\right), x_{n+1}\right] .
$$

Since, by [9], $J$ and $R$ satisfy the same differential identities, then we have that $R$ satisfies $F\left(f\left(x_{1}, \ldots, x_{n}\right)\right) \in C$. Let $S$ be the additive subgroup generated by the subset

$$
\left\{f\left(r_{1}, \ldots, r_{n}\right) \mid r_{1}, \ldots, r_{n} \in R\right\} \text {. }
$$

$S$ is a Lie ideal of $R$; indeed for any $r \in R, y_{1}, \ldots, y_{n} \in R$ one has

$$
\left[r, f\left(y_{1}, \ldots, y_{n}\right)\right]=\sum_{i} f\left(y_{1}, \ldots,\left[r, y_{i}\right], \ldots, y_{n}\right) \in S .
$$

If $S$ is noncommutative then, by [10, pages 4-5], there exists a nonzero two-sided ideal $I$ of $R$ such that $0 \neq[I, R] \subseteq S$. In this case it is easy to see that $F\left(\left[r_{1}, r_{2}\right]\right) \in C$, for all $r_{1}, r_{2} \in I$. In particular $[F(u), u]=0$ for all $u \in[I, I]$. Since $[I, I]$ is a noncentral Lie ideal of $R$, it follows easily that $F$ must be zero (see, e.g., Theorem 3.3 in [11]).

Hence we may consider it the only case when $S$ is commutative.

Thus $\left[f\left(x_{1}, \ldots, x_{n}\right), f\left(y_{1}, \ldots, y_{n}\right)\right]$ is an identity in $R$. This means that there exist a field $K$ and a positive integer $m$ such that $\left[f\left(x_{1}, \ldots, x_{n}\right), f\left(y_{1}, \ldots, y_{n}\right)\right]$ is also an identity in $M_{m}(K)$. If $m=1, R$ is commutative, thus we suppose $m \geq 2$.

Since $f\left(x_{1}, \ldots, x_{n}\right)$ is not central valued on $R$, there exist $r_{1}, \ldots, r_{n} \in M_{m}(K)$ such that $f\left(r_{1}, \ldots, r_{n}\right)=a \notin Z(R)$, so that $\left[a, f\left(y_{1}, \ldots, y_{n}\right)\right]$ is also a generalized identity in $M_{m}(K)$. By a result of Lee (see [12], Theorem), we have the contradiction that $f\left(x_{1}, \ldots, x_{n}\right)$ is central valued on $R$.

Lemma 8. Let $R$ be a 2-torsion free primitive ring which is isomorphic to a dense ring of linear transformations of a vector space $V$ over the field $C$ such that $\operatorname{dim}_{C} V \geq 3$, and let $a, b \in R$, $\gamma \in C, m, n \geq 1$, different integers. If for any $x, y \in R$, $\gamma[x, y]^{m}+a[x, y]^{m}+[x, y]^{m} b=a[x, y]^{n}+[x, y]^{n} b$, then $\gamma=0$, $a+b=0$ and one of the following holds:

(a) $a, b \in C$;

(b) for all $x_{1}, x_{2} \in R$ there exists $\alpha \in C$ such that $\left[x_{1}, x_{2}\right]^{m}=\left[x_{1}, x_{2}\right]^{n}+\alpha$.

Proof. Let $0 \neq v \in V$ such that $\{v, v a\}$ are linearly $C$ independent. Since $\operatorname{dim}_{C} V \geq 3$, there exists $w \in V$ such that $\{v, v a, w\}$ are linearly $C$-independent. By the density of $R$ there exist $x, y \in R$ such that

$$
\begin{gathered}
\operatorname{vax}=w, \quad v a y=0, \quad v x=w, \\
v y=0, \quad w y=v
\end{gathered}
$$

so that $v[x, y]=v, v a[x, y]=0$ and by the hypothesis

$$
\begin{gathered}
0=v\left(\gamma[x, y]^{m}+a[x, y]^{m}+[x, y]^{m} b\right. \\
\left.-a[x, y]^{n}-[x, y]^{n} b\right)=\gamma v
\end{gathered}
$$

which implies $\gamma=0$. Thus $a\left([x, y]^{m}-[x, y]^{n}\right)+\left([x, y]^{m}-\right.$ $\left.[x, y]^{n}\right) b=0$ for all $x, y \in R$ and by Lemma 7 we get the required conclusions.

Assume now that, for any $v \in V,\{v, v a\}$ are linearly $C$ dependent. In this case standard arguments show that $a \in C$; hence $[x, y]^{m}(\gamma+a+b)=[x, y]^{n}(a+b)$ and by Lemma 6 we get $\gamma+a+b=a+b$; that is, $\gamma=0$ and $a=-b \in C$.

Lemma 9. Let $R$ be a 2-torsion free primitive ring which is isomorphic to a dense ring of linear transformations of a vector space $V$ over the field $C$ such that $\operatorname{dim}_{C} V \geq 3$, and let $a, b, c, q \in$ $R$ and $m, n \geq 1$ be such that $a[x, y]^{m}+[x, y]^{m} b=c[x, y]^{n}+$ $[x, y]^{n} q$, for all $x, y \in R$. Then one of the following holds:

(a) $a, b, c, q \in C, a+b=0$ and $c+q=0$;

(b) $a-c=q-b \in C, a+b=c+q=0$ and for all $x_{1}, x_{2} \in R$ there exists $\alpha \in C$ such that $\left[x_{1}, x_{2}\right]^{m}=\left[x_{1}, x_{2}\right]^{n}+\alpha$;

(c) $a-c=q-b \in C$ and $\left[x_{1}, x_{2}\right]^{m}=\left[x_{1}, x_{2}\right]^{n}$ for all $x_{1}, x_{2} \in R$.

Proof. Suppose first that $\operatorname{dim}_{C} V=t$ is finite. In this case $R \cong$ $M_{t}(C)$, the ring of all $t \times t$ matrices over $C$ with $t \geq 3$. Let $e_{i j}$ be the usual matrix unit and denote $a=\sum a_{r s} e_{r s}, c=\sum c_{r s} e_{r s}$, with $a_{r s}, c_{r s} \in C$. Fix $i \neq j$ and choose $[x, y]=\left[e_{i j}, e_{j i}\right]=e_{i i}-$ $e_{j j}$. By the hypothesis

$$
a\left(e_{i i}-e_{j j}\right)^{m}+\left(e_{i i}-e_{j j}\right)^{m} b=c\left(e_{i i}-e_{j j}\right)^{n}+\left(e_{i i}-e_{j j}\right)^{n} q
$$

and left multiplying by $e_{k k}$, for any $k \neq i, j$, it follows that $a_{k i}-c_{k i}=0$. This means that the matrix $a-c$ is diagonal. Let now $\varphi$ be any automorpism of $R$ and note that $\varphi(a)[x, y]^{m}+$ $[x, y]^{m} \varphi(b)=\varphi(c)[x, y]^{n}+[x, y]^{n} \varphi(q)$, for all $x, y \in R$. Therefore $\varphi(a-c)$ must be a diagonal matrix. In particular, for any $i \neq j,\left(1+e_{i j}\right)(a-c)\left(1-e_{i j}\right)$ must be a diagonal matrix. By easy computation it follows that $a_{j j}-c_{j j}=a_{i i}-c_{i i}$; that is, $a-c=\alpha \in Z(R)$. Analogously one can prove that $b-q=\beta \in Z(R)$.

Therefore we have that

$$
(\alpha+\beta)[x, y]^{m}+c[x, y]^{m}+[x, y]^{m} q=c[x, y]^{n}+[x, y]^{n} q .
$$

Suppose now that $\operatorname{dim}_{C} V=\infty$. By Lemma 2 in [13], $R$ satisfies the following generalized identity $a x^{m}+x^{m} b-c x^{n}-$ $x^{n} q$. Let $e \in H=\operatorname{soc}(R)$ be any idempotent minimal element 
and recall that $H$ is generated by such idempotent elements. Since $H$ satisfies $a x^{m}+x^{m} b-c x^{n}-x^{n} q$, then in particular $a e+e b-c e-e q=0$. Thus we can easily obtain both $(1-e)(a-$ $c) e=0$ and $e(b-q)(1-e)=0$, which imply $[a-c, e]=0$ and $[b-q, e]=0$; that is, $[a-c, H]=0$ and $[b-q, H]=0$. Also in this case there exist $\alpha, \beta \in C$ such that $a-c=\alpha$ and $b-q=\beta$. Therefore in any case Identity (9) holds and the conclusion follows as an application of Lemma 8.

Lemma 10. Let $R$ be a 2-torsion free prime ring, $a, b, c, q \in R$, and let $m, n \geq 1$ be such that $a[x, y]^{m}+[x, y]^{m} b=c[x, y]^{n}+$ $[x, y]^{n} q$, for all $x, y \in R$. Then either $R$ satisfies $s_{4}$, the standard identity of degree 4, or one of the following holds:

(a) $a, b, c, q \in C, a+b=0$ and $c+q=0$;

(b) $a-c=q-b \in C, a+b=c+q=0$ and for all $x_{1}, x_{2} \in R$ there exists $\alpha \in C$ such that $\left[x_{1}, x_{2}\right]^{m}=\left[x_{1}, x_{2}\right]^{n}+\alpha$;

(c) $a-c=q-b \in C$ and $\left[x_{1}, x_{2}\right]^{m}=\left[x_{1}, x_{2}\right]^{n}$ for all $x_{1}, x_{2} \in R$.

Proof. Firstly assume that $R$ does not satisfy any nontrivial generalized polynomial identity. In light of [14] and by our assumption, it follows that both

$$
\begin{aligned}
& a[x, y]^{m}-c[x, y]^{n}, \\
& {[x, y]^{m} b-[x, y]^{n} q}
\end{aligned}
$$

are trivial generalized polynomial identities of $R$. This means that $a, b, c, q \in C$, so that $(a+b)[x, y]^{m}-(c+q)[x, y]^{n}$ is a trivial generalized polynomial identity for $R$.

Hence $a+b=0$ and $c+q=0$.

Consider now the case that $R$ satisfies some nontrivial generalized polynomial identity. By Theorem 3 in [15] it follows that $S=R C$ is a primitive ring with $\operatorname{soc}(R) \neq 0$, where $C=Z(U)$ is the extended centroid of $R$, and the Utumi quotient ring $U$ is a $C$-algebra centrally closed. Since $R$ and $U$ satisfy the same generalized polynomial identities (see [14]), without loss of generality, we may replace $R$ by $U$ and $Z(R)$ by $C$ and $R$ is a $C$-algebra centrally closed. Then $R$ is a dense ring of linear transformations of a vector space $V$ over $C$. In case $\operatorname{dim}_{C} V \leq 2$, then $R$ satisfies the standard identity $s_{4}$. In case $\operatorname{dim}_{C} V \geq 3$ we may apply Lemma 9 . In any case we are done.

We consider now the more general situation.

Proof of Theorem 1. In all that follows we assume that $R$ does not satisfy $s_{4}$; if not we are done.

Since any generalized derivation $H$ of $R$ can be implicitly assumed to be defined on the whole $U$ and assumes the form $H(x)=c x+h(x)$ for some $c \in U$ and $h$ a derivation on $U$, we may assume that there exist $a, b \in U$ and $d, \delta$ derivations on $U$ such that

$$
F(x)=a x+d(x), \quad G(x)=b x+\delta(x) .
$$

Since $R$ and $U$ satisfy the same generalized polynomial identities [14] as well as the same differential identities [9], then, without loss of generality, to prove our results we may assume that

$$
F\left([x, y]^{m}\right)=G\left([x, y]^{n}\right) \quad \forall x, y \in U,
$$

where $d, \delta$ are derivations on $U$. We divide the proof into 3 cases.

Case 1. Let $d(x)=[c, x]$ and $\delta(x)=[q, x]$ be both inner derivations in $U$, so that $F(x)=a x+[c, x]=(a+c) x-x c$ and $G(x)=b x+[q, x]=(b+q) x-x q$, for suitable elements $c, q \in U$. Thus we have $(a+c)[x, y]^{m}+[x, y]^{m}(-c)=(b+$ q) $[x, y]^{n}+[x, y]^{n}(-q)$, for all $x, y \in U$. In this case we apply Lemma 10 and we have that either $R$ satisfies $s_{4}$ or one of the following holds:

(a) $a=b=0$ and $d=\delta=0$; that is, $F=G=0$;

(b) $a=b=0, c-q \in C$, and $d=\delta=F=G$; that is, $F=G$ is the inner derivation induced by the element $c$; moreover for all $x_{1}, x_{2} \in R$ there exists $\alpha \in C$ such that $\left[x_{1}, x_{2}\right]^{m}=\left[x_{1}, x_{2}\right]^{n}+\alpha$;

(c) $\left[x_{1}, x_{2}\right]^{m}=\left[x_{1}, x_{2}\right]^{n}$, for all $x_{1}, x_{2} \in R, q-c \in C$ (i.e., $d=\delta)$; moreover $a=b+\beta$ for a suitable $\beta \in C$. In this case, our assumption implies that $\beta\left[x_{1}, x_{2}\right]^{m}=0$ for all $x_{1}, x_{2} \in R$, which means $\beta=0$, since $R$ is not commutative. Therefore $a=b$ and $F=G$.

Case 2. Assume that $d$ and $\delta$ are $C$-independent modulo $U$ inner derivations.

By the main assumption, $U$ satisfies

$$
\begin{aligned}
& a[x, y]^{n}+\sum_{i+j=m-1}[x, y]^{i}([d(x), y]+[x, d(y)])[x, y]^{j} \\
& \quad=b[x, y]^{n}+\sum_{i+j=n-1}[x, y]^{i}([\delta(x), y]+[x, \delta(y)])[x, y]^{j} .
\end{aligned}
$$

Then by the result in [16], we have that $U$ satisfies

$$
\begin{aligned}
& a[x, y]^{n}+\sum_{i+j=m-1}[x, y]^{i}\left(\left[x_{1}, y\right]+\left[x, x_{2}\right]\right)[x, y]^{j} \\
& \quad=b[x, y]^{n}+\sum_{i+j=n-1}[x, y]^{i}\left(\left[x_{3}, y\right]+\left[x, x_{4}\right]\right)[x, y]^{j} .
\end{aligned}
$$

In particular $U$ satisfies the blended component

$$
\sum_{i+j=m-1}[x, y]^{i}\left[x_{1}, y, x, y\right]^{j} .
$$

Thus $U$ is a PI-ring and there exists $M_{t}(C)$ satisfying (15). For $x=e_{12}, y=e_{21}$, and $x_{1}=e_{32}$ we have the contradiction $e_{31}=0$.

Case 3. Assume finally that $d$ and $\delta$ are not both inner derivations (otherwise we are done by Case 1) such that $d$ and $\delta$ are $C$-dependent modulo $U$-inner derivations. Without loss of generality say $d=\alpha \delta+a d_{q}$; that is, $d(x)=\alpha \delta(x)+[q, x]$, for suitable $\alpha \in C$ and $q \in U$. Under this assumption, we prove 
that a number of contradictions follow. We may assume the following:

(i) $d, \delta$ are both nonzero derivations of $R$. In fact, if either $d$ or $\delta$ is zero then both $F$ and $G$ are inner generalized derivations of $R$, which is a contradiction;

(ii) $\delta$ is not an inner derivation, if not both $F$ and $G$ are inner generalized derivations of $R$, which is again a contradiction.

Suppose first that $\alpha=0$; then $d(x)=[q, x]$. Thus $U$ satisfies

$$
a[x, y]^{m}+\left[q,[x, y]^{m}\right]=b[x, y]^{n}+\delta\left([x, y]^{n}\right)
$$

that is,

$$
\begin{aligned}
& (a+q)[x, y]^{m}+[x, y]^{m}(-q) \\
& =b[x, y]^{n}+\sum_{i+j=n-1}[x, y]^{i}([\delta(x), y]+[x, \delta(y)])[x, y]^{j} .
\end{aligned}
$$

By [16] it follows that $U$ satisfies

$$
\begin{aligned}
& (a+q)[x, y]^{m}+[x, y]^{m}(-q) \\
& =b[x, y]^{n}+\sum_{i+j=n-1}[x, y]^{i}\left(\left[x_{1}, y\right]+\left[x, x_{2}\right]\right)[x, y]^{j}
\end{aligned}
$$

and in particular $U$ satisfies the blended component

$$
\sum_{i+j=n-1}[x, y]^{i}\left[x_{1}, y\right][x, y]^{j} .
$$

Therefore $U$ is a PI-ring, so that there exists a field $K$ such that $U$ and the matrix $\operatorname{ring} M_{t}(K)$ satisfy the same polynomial identities. Moreover, we may assume $t \geq 3$, since $U$ does not satisfy $s_{4}$. Notice that if we choose $x=e_{12}, y=e_{21}$, and $x_{1}=$ $e_{32}$ then the contradiction $e_{31}=0$ follows.

Then assume $\alpha \neq 0$. Thus $U$ satisfies

$$
\begin{gathered}
a[x, y]^{m}+\alpha \delta\left([x, y]^{m}\right)+\left[q,[x, y]^{m}\right] \\
=b[x, y]^{n}+\delta\left([x, y]^{n}\right)
\end{gathered}
$$

that is,

$$
\begin{aligned}
& (a+q)[x, y]^{m}+[x, y]^{m}(-q) \\
& \quad+\alpha \sum_{i+j=m-1}[x, y]^{i}([\delta(x), y]+[x, \delta(y)])[x, y]^{j} \\
& =b[x, y]^{n}+\sum_{i+j=n-1}[x, y]^{i}([\delta(x), y]+[x, \delta(y)])[x, y]^{j}
\end{aligned}
$$

By [16] it follows that $U$ satisfies

$$
\begin{aligned}
& (a+q)[x, y]^{m}+[x, y]^{m}(-q) \\
& \quad+\alpha \sum_{i+j=m-1}[x, y]^{i}\left(\left[x_{1}, y\right]+\left[x, x_{2}\right]\right)[x, y]^{j} \\
& =b[x, y]^{n}+\sum_{i+j=n-1}[x, y]^{i}\left(\left[x_{1}, y\right]+\left[x, x_{2}\right]\right)[x, y]^{j}
\end{aligned}
$$

and in particular $U$ satisfies both

$$
\begin{aligned}
& (a+q)[x, y]^{m}+[x, y]^{m}(-q)=b[x, y]^{n}, \\
\alpha & \sum_{i+j=m-1}[x, y]^{i}\left(\left[x_{1}, y\right]+\left[x, x_{2}\right]\right)[x, y]^{j} \\
= & \sum_{i+j=n-1}[x, y]^{i}\left(\left[x_{1}, y\right]+\left[x, x_{2}\right]\right)[x, y]^{j} .
\end{aligned}
$$

As above, $U$ is a PI-ring, so that there exists a field $K$ such that $U$ and the matrix ring $M_{t}(K)$ satisfy (24). Since we may assume that $t \geq 3$, then for $x=e_{12}, y=e_{21}, x_{1}=e_{32}$, and $x_{2}=0$ in (24) we get $\alpha e_{31}=e_{31}$; that is, $\alpha=1$. Moreover, by (23) and using Lemma 10 we have in any case $q \in C$, that is, $d(x)=\delta(x)$, and one of the following holds.

(a) $u^{m}=u^{n}$ for all $u \in[U, U]$ and there exists $\beta \in C$ such that $b=a+\beta$. In this case we have $F(x)=a x+d(x)$ and $G(x)=(a+\beta) x+d(x)$ for all $x \in U$. By the main assumption we also get $a u^{m}+d\left(u^{m}\right)=a u^{m}+$ $\beta u^{m}+d\left(u^{m}\right)$, for all $u \in[U, U]$. Hence $\beta u^{m}=0$, which implies $\beta=0$ and $F=G$.

(b) $a=b=0$ and $F=d=\delta=G$. This implies $d\left(u^{m}-\right.$ $\left.u^{n}\right)=0$, for all $u \in[U, U]$. Since $d \neq 0$, by Lemma 7 we have that for all $u \in[U, U]$ there exists $\beta_{u} \in C$ such that $u^{m}-u^{n}=\beta_{u}$. Without loss of generality we consider $n>m$ and let $\alpha$ be any nonzero element of $C$. Denote $v=[x, y] \in[U, U]$ and $u=\left[\alpha^{-m} x, y\right]=$ $\alpha^{-m} v \in[U, U]$. Thus there exist $\beta_{u}, \beta_{v} \in C$ such that $v^{m}=v^{n}+\beta_{v}$ and $u^{m}=u^{n}+\beta_{u}$. Hence $\alpha v^{m}=\alpha^{n-m} v^{n}+$ $\beta_{u}$ implying

$$
\alpha v^{n}+\alpha \beta_{v}=\alpha^{n-m} v^{n}+\beta_{u} .
$$

In other words, for all $\alpha \in C$ and for all $v \in[U, U]$, we have $\left(\alpha-\alpha^{n-m}\right) v^{n} \in C$. In case $\alpha \neq \alpha^{n-m}$ then $v^{n} \in C$ for all $v \in[U, U]$ and as above there exists a field $K$ such that $U$ and the matrix ring $M_{t}(K)$ satisfy $\left[[x, y]^{n}, z\right]=0$. Since we may assume that $t \geq 3$, a contradiction follows easily. Thus $\alpha=\alpha^{n-m}$ for any $\alpha \in C$. In this last case and by (25), it follows that $\alpha \beta_{v}=\beta_{u}$, for any $\alpha \neq 0$. Hence, for $0 \neq \alpha_{1} \neq \alpha_{2} \in C$, we get $\alpha_{1} \beta_{v}=\alpha_{2} \beta_{v}$; that is, $\beta_{v}=0$ for any choice of $v \in[U, U]$, which means $v^{m}=v^{n}$ for all $v \in[U, U]$.

Corollary 11. Let $R$ be a 2-torsion free prime ring and let $L$ be a noncentral Lie ideal of $R$, and let $F: R \rightarrow R$ and $G: R \rightarrow R$ be two generalized derivations associated with derivations $d$ : $R \rightarrow R$ and $\delta: R \rightarrow R$, respectively. Suppose that there exist $m, n \geq 1$ integers such that $F\left(u^{m}\right)=G\left(u^{n}\right)$ for all $u \in L$. Then either $R$ satisfies the standard identity $s_{4}$ or one of the following holds: 
(a) $F=G$ is an inner ordinary derivation of $R$ and for all $x_{1}, x_{2} \in R$ there exists $\alpha \in C$ such that $\left[x_{1}, x_{2}\right]^{m}=$ $\left[x_{1}, x_{2}\right]^{n}+\alpha$

(b) $F=G$ and $\left[x_{1}, x_{2}\right]^{m}=\left[x_{1}, x_{2}\right]^{n}$, for all $x_{1}, x_{2} \in R$.

Proof. Since $L$ is not central in $R$, then by [10, pages 4-5] there exists a nonzero two-sided ideal $I$ of $R$ such that $[I, I] \subseteq L$. Therefore $F\left([x, y]^{m}\right)=G\left([x, y]^{n}\right)$ for all $x, y \in I$. As above we write

$$
F(x)=a x+d(x), \quad G(x)=b x+\delta(x)
$$

for suitable $a, b \in U$ and $d, \delta$ derivations of $R$. Thus $I$ satisfies the differential identity

$$
a[x, y]^{m}+d\left([x, y]^{m}\right)-b[x, y]^{n}-\delta\left([x, y]^{n}\right) .
$$

Since $I, R$, and $U$ satisfy the same differential identities (see [9]), then (27) is satisfied by $U$. Hence we conclude by Theorem 1.

Proof of Theorem 2. Firstly we notice that for $u=v$ we have $F\left(u^{m(p+q)}\right)=G\left(u^{n(r+s)}\right)$, for all $u \in L$. By Corollary 11 we have that $R$ is a PI-ring and, if assumed that $R$ does not satisfy $s_{4}$, then one has $F=G$. As remarked above, there exists an ideal $I$ of $R$ such that $[I, I] \subseteq L$; therefore

$$
F\left(\left(u^{p} v^{q}\right)^{m}-\left(v^{r} u^{s}\right)^{n}\right)=0
$$

for all $u, v \in[I, I]$. Since $U$ and $I$ satisfy the same differential identities, we also have that (28) is satisfied by $U$. By Lemma 7 , and since $F \neq 0,\left(u^{p} v^{q}\right)^{m}-\left(v^{r} u^{s}\right)^{n}$ is central for all $u, v \in$ $[U, U]$, moreover there exists a field $K$ such that $M_{t}(K)$ and $U$ satisfy the same polynomial identities. Of course we assume that $t \geq 3$, since $U$ does not satisfy $s_{4}$. Fix $u=\left[e_{12}, e_{21}+e_{31}\right]=$ $e_{11}-e_{22}-e_{32}$ and $v=\left[e_{12}, e_{21}\right]=e_{11}-e_{22}$.

Thus

$$
\begin{gathered}
\left(u^{p} v^{q}\right)^{m}=e_{11}+\alpha e_{22}+\beta e_{32} \\
\left(v^{r} u^{s}\right)^{n}=e_{11}+\gamma e_{22}
\end{gathered}
$$

where $\alpha, \beta, \gamma \in\{+1,-1\}$. Hence

$$
\left(u^{p} v^{q}\right)^{m}-\left(v^{r} u^{s}\right)^{n}=\beta e_{32} \notin Z\left(M_{t}(K)\right),
$$

which is a contradiction.

Corollary 12. Let $R$ be a 2-torsion free prime ring, $U$ its Utumi quotient ring, $C$ its extended centroid, $L$ noncentral Lie ideal of $R$, and $F: R \rightarrow R$ and $G: R \rightarrow R$ two generalized derivations associated with derivations $d: R \rightarrow R$ and $\delta:$ $R \rightarrow R$, respectively. Suppose that there exists $n \geq 1$ integer such that $F\left((u v)^{n}\right)=G\left((v u)^{n}\right)$ for all $u, v \in L$. Then either $F=G=0$ or $R$ satisfies the standard identity $s_{4}$ and $F-G$ is an ordinary derivation of $R$.

Proof. For $u=v \in L$ in our main assumption we get $F\left(u^{2 n}\right)=$ $G\left(u^{2 n}\right)$; moreover by applying Theorem 2 it follows that $R$ must satisfy the standard identity $s_{4}$.
Therefore $U \cong M_{2}(C)$ and we may assume that $R \subseteq$ $M_{2}(C)$, the $2 \times 2$ matrix ring over $C$. Since $L$ is not central and $R$ is 2-torsion free, as remarked above, it follows that $[R, R] \subseteq L$. Hence we have that $F\left(\left[x_{1}, x_{2}\right]^{2 n}\right)=G\left(\left[x_{1}, x_{2}\right]^{2 n}\right)$ is satisfied by $R$. Let $a, b \in U$ such that $F(x)=a x+d(x)$ and $G(x)=b x+\delta(x)$, for all $x \in R$; then $R$ satisfies the generalized differential identity

$$
(a-b)\left[x_{1}, x_{2}\right]^{2 n}+(d-\delta)\left(\left[x_{1}, x_{2}\right]^{2 n}\right) .
$$

Firstly notice that in case $F=0$ then $R$ satisfies $G\left(\left[x_{1}, x_{2}\right]\left[y_{1}, y_{2}\right]^{n}\right)$ and by Lemma 7 it follows that $G=0$ unless $\left[x_{1}, x_{2}\right]\left[y_{1}, y_{2}\right]$ is central valued on $R$. In the latter case for $\left[x_{1}, x_{2}\right]=e_{12}$ and $\left[y_{1}, y_{2}\right]=e_{21}$ a contradiction follows.

Thus we assume both $F \neq 0$ and $G \neq 0$ and denote $H(x)=$ $d(x)-\delta(x)$ (of course $H$ is a derivation of $R$ ). Here we apply again Kharchenko's theory, using the fact that $\left[x_{1}, x_{2}\right]^{2 n}$ is central valued on $R$, and we prove that $a=b$. To do this, we divide the proof into two cases.

Case 1. Let $H(x)=[c, x]$ be the inner derivations in $R$. Thus we have that $(a-b)[x, y]^{2 n}=0$, for all $x, y \in R$, which implies $a=b$.

Case 2. Assume that $H$ is not inner. By Kharchenko's theory in [16], and since $R$ satisfies

$$
\begin{aligned}
& (a-b)\left[x_{1}, x_{2}\right]^{2 n} \\
& +\sum_{i+j=2 n-1}\left[x_{1}, x_{2}\right]^{i}\left(\left[H\left(x_{1}\right), x_{2}\right]+\left[x_{1}, H\left(x_{2}\right)\right]\right)\left[x_{1}, x_{2}\right]^{j},
\end{aligned}
$$

then $R$ satisfies

$$
\begin{aligned}
& (a-b)\left[x_{1}, x_{2}\right]^{2 n} \\
& +\sum_{i+j=2 n-1}\left[x_{1}, x_{2}\right]^{i}\left(\left[y_{1}, x_{2}\right]+\left[x_{1}, y_{2}\right]\right)\left[x_{1}, x_{2}\right]^{j}
\end{aligned}
$$

in particular $(a-b)\left[x_{1}, x_{2}\right]^{2 n}$ is a generalized identity for $R$, which implies again that $a=b$.

Corollary 13. Let $R$ be a 2-torsion free prime ring, $U$ its Utumi quotient ring, $C$ its extended centroid, and $F: R \rightarrow R$ and $G: R \rightarrow R$ two generalized derivations associated with derivations $d: R \rightarrow R$ and $\delta: R \rightarrow R$, respectively. Suppose that there exists $n \geq 1$ integer such that $F\left((u v)^{n}\right)=G\left((v u)^{n}\right)$ for all $u, v \in R$. Then either $F=G=0$ or $R$ is commutative.

Proof. By Corollary 12, if we assume that $F \neq 0$ and $G \neq 0$, it follows that $R \subseteq M_{2}(C)$ and $F-G=H$ is ordinary derivation of $R$. In particular $H\left(x^{2}\right)=0$ for all $x \in R$ and by Lemma 7 it follows that either $H=0$ or $x^{2} \in Z(R)$, for all $x \in R$.

In case $H=0$, then $F=G$ and $F\left((u v)^{n}-(v u)^{n}\right)=0$ for all $u, v \in R$. Again by Lemma 7 , since $F \neq 0$, one has $(u v)^{n}-$ $(v u)^{n} \in Z(R)$; in particular for $u=e_{11}$ and $v=e_{11}+e_{12}$, it follows the contradiction $e_{12} \in Z(R)$. Therefore $R$ must be commutative.

Let $H \neq 0$, so that $x^{2} \in Z(R)$, for all $x \in R$ follows by the previous argument. Thus $R$ is commutative and by the 
main assumption it follows $H\left((u v)^{n}\right)=0$. Once again by Lemma 7 , since $H \neq 0$, it follows $(u v)^{n} \in Z(R)$, which leads to a contradiction, as remarked above.

An easy consequence of Corollary 13 is the following.

Corollary 14. Let $R$ be a 2-torsion free prime ring, $U$ its Utumi quotient ring, $C$ its extended centroid, and $F: R \rightarrow R$ a generalized derivation of $R$. Suppose that there exists $n \geq 1$ integer such that $F\left((u v)^{n}\right)=0$ for all $u, v \in R$. Then either $F=0$ or $R$ is commutative.

\section{Results in Semiprime Rings}

In order to prove the main result of this section we will make use of the following facts.

Remark 15. Let $R$ be a semiprime ring and let $F$ be a generalized derivation of $R$ associated with derivation $d$. If $F(R)=0$, then $d(R)=0$.

Claim 1 (see [17, Proposition 2.5.1]). Any derivation of a semiprime ring $R$ can be uniquely extended to a derivation of its left Utumi quotient ring $U$, and so any derivation of $R$ can be defined on the whole $U$.

Claim 2 (see [18, page 38]). If $R$ is semiprime then so is its left Utumi quotient ring.

The extended centroid $C$ of a semiprime ring coincides with the center of its left Utumi quotient ring.

Claim 3 (see [18, p. 42]). Let $B$ be the set of all the idempotents in $C$, the extended centroid of $R$. Assume that $R$ is B-algebra orthogonal complete. For any maximal ideal $P$ of $B, P R$ forms a maximal prime ideal of $R$, which is invariant under any derivation of $R$.

We are now ready to prove Theorems 3 and 4 .

Proof of Theorem 3. Let $a, b \in U$ and $d, \delta$ derivations of $U$ be such that $F(x)=a x+d(x)$ and $G(x)=b x+\delta(x)$, for all $x \in R$. By Claim 2, $Z(U)=C$, and by Claim $1 d$ and $\delta$ can be uniquely defined on the whole $U$. Since $U$ and $R$ satisfy the same generalized differential identities, then $F\left((x y)^{n}\right)=$ $G\left((y x)^{n}\right)$, for all $x, y \in[U, U]$. Let $B$ be the complete Boolean algebra of idempotents in $C$ and $M$ any maximal ideal of $B$.

Since $U$ is $B$-algebra orthogonal complete (see [18, page 42, (2) of Fact 1]), by Claim 3, MU is a prime ideal of $U$, which is both $d$-invariant and $\delta$-invariant. Let $\bar{d}$ and $\bar{\delta}$ be the derivations induced, respectively, by $d$ and $\delta$ on $\bar{U}=U / M U$ and denote $\bar{F}(\bar{x})=\overline{a x}+\bar{d}(\bar{x}), \bar{G}(\bar{x})=\bar{b} \bar{x}+\bar{\delta}(\bar{x})$. For any $\bar{x}, \bar{y} \in[\bar{U}, \bar{U}], \bar{F}\left((\overline{x y})^{n}\right)=\bar{G}\left((\overline{y x})^{n}\right)$. In particular $\bar{U}$ is a prime ring and so, by Corollary 12 , either $\bar{F}=0$ and $\bar{G}=0$ in $\bar{U}$ or $\bar{U}$ satisfies $s_{4}$. This implies that, for any maximal ideal $M$ of $B, F(U) \subseteq M U$ and $G(U) \subseteq M U$ or $s_{4}\left(x_{1}, x_{2}, x_{3}, x_{4}\right) \subseteq M U$, for all $x_{1}, x_{2}, x_{3}, x_{4} \in U$. In any case $F(U) s_{4}\left(x_{1}, x_{2}, x_{3}, x_{4}\right) \subseteq$ $\bigcap_{M} M U=0$ and also $G(U) s_{4}\left(x_{1}, x_{2}, x_{3}, x_{4}\right) \subseteq \bigcap_{M} M U=$ 0 . From [17, Chapter 3] there exists a central idempotent element $e$ of $U$, such that $F(e U)=0, G(e U)=0$ and $(1-e) U$ satisfies $s_{4}$.

Proof of Theorem 4. Here we repeat the same argument above. Let $a, b \in U$ such that $F(x)=a x+d(x)$ and $G(x)=b x+\delta(x)$. Let $B$ be the complete Boolean algebra of idempotents in $C$ and $M$ any maximal ideal of $B$.

Since $U$ is $B$-algebra orthogonal complete (see $[18, \mathrm{p}$. 42, (2) of Fact 1]), by Claim 3, $M U$ is a prime ideal of $U$, which is both $d$-invariant and $\delta$-invariant. Let $\bar{d}$ and $\bar{\delta}$ be two derivations induced, respectively, by $d$ and $\delta$ on $\bar{U}=U / M U$ and denote $\bar{F}(\bar{x})=\overline{a x}+\bar{d}(\bar{x}), \bar{G}(\bar{x})=\bar{b} \bar{x}+\bar{\delta}(\bar{x})$. For any $\bar{x}, \bar{y} \in[\bar{U}, \bar{U}], \bar{F}\left((\overline{x y})^{n}\right)=\bar{G}\left((\overline{y x})^{n}\right)$. Since $\bar{U}$ is a prime ring so, by Corollary 13 , either $\bar{F}=0$ and $\bar{G}=0$ in $\bar{U}$ or $\bar{U}$ is commutative. Moreover, if $\bar{F}=\overline{0}$ and $\bar{G}=\overline{0}$ in $\bar{U}$ then, by Remark 15, it follows that $\bar{d}=\overline{0}$ and $\bar{\delta}=\overline{0}$ in $\bar{U}$.

This implies that, for any maximal ideal $M$ of $B$, one of the following holds:

(a) either $F(U) \subseteq M U, d(U) \subseteq M U, G(U) \subseteq M U$ and $\delta(U) \subseteq M U$;

(b) or $[U, U] \subseteq M U$.

In any case $[F(U), U] \subseteq \bigcap M U=0$ and $[d(U), U] \subseteq$ $\bigcap M U=0$ imply that $[a U, U]=0$. Analogously $[G(U), U] \subseteq$ $\bigcap M U=0$ and $[\delta(U), U] \subseteq \bigcap M U=0$ implying that $[b U, U]=0$.

As mentioned above it follows that there exists a central idempotent element $e$ in $U$ such that, on the direct sum decomposition $e U \oplus(1-e) U, F$ and $G$ vanish identically on $e U$ and the ring $(1-e) U$ is commutative.

Moreover, $\left[a U^{2}, U\right]=(0)$ implies $a U[U, U]=(0)$; that is, both $U a U^{2}[U, U]=(0)$ and $a U U^{2}[U, U]=(0)$. Therefore $[a, U] U^{2}[a, U]=(0)$ and, by the semiprimeness of $U$, it follows $[a, U]=(0)$; that is, $a \in C$. Analogously one can prove that $b \in C$.

On the other hand, since by Theorem 3 in [9] $U$ and $R$ satisfy the same differential identities, then $[d(R), R]=0$ and $[\delta(R), R]=0$, which imply that $d(R) \subseteq Z(R), \delta(R) \subseteq Z(R)$, and $R$ contains some nonzero central ideals, unless when $d(R)=0$ and $\delta(R)=0$.

In the last case, $\lambda(u v)^{n}=\mu(v u)^{n}$ for all $u, v \in R$, and in particular $\lambda(u)^{2 n}=\mu(u)^{2 n}$ for all $u \in R$. The semiprimeness of $R$ forces $\lambda=\mu \neq 0($ since $F \neq 0)$, so that $(u v)^{n}=(v u)^{n}$ for all $u, v \in R$. Finally by Theorem 1 in [19], the commutativity of $R$ follows which is a contradiction.

\section{Conflict of Interests}

The authors declare that there is no conflict of interests regarding the publication of this paper.

\section{References}

[1] T.-K. Lee, "Generalized derivations of left faithful rings," Communications in Algebra, vol. 27, no. 8, pp. 4057-4073, 1999.

[2] J. Bergen, "Automorphisms with unipotent values," Rendiconti del Circolo Matematico di Palermo, vol. 31, no. 2, pp. 226-232, 1982. 
[3] M. N. Daif and H. E. Bell, "Remarks on derivations on semiprime rings," International Journal of Mathematics and Mathematical Sciences, vol. 15, no. 1, pp. 205-206, 1992.

[4] M. Hongan, "A note on semiprime rings with derivation," International Journal of Mathematics and Mathematical Sciences, vol. 20, no. 2, pp. 413-415, 1997.

[5] M. Ashraf and S. Ali, "On left multipliers and the commutativity of prime rings," Demonstratio Mathematica, vol. 41, no. 4, pp. 763-771, 2008.

[6] S. Ali and S. Huang, "On left $\alpha$-multipliers and commutativity of semiprime rings," Communications of the Korean Mathematical Society, vol. 27, no. 1, pp. 69-76, 2012.

[7] S. Andima and H. Pajoohesh, "Commutativity of rings with derivations," Acta Mathematica Hungarica, vol. 128, no. 1-2, pp. $1-14,2010$.

[8] C.-L. Chuang and T.-K. Lee, "Rings with annihilator conditions on multilinear polynomials," Chinese Journal of Mathematics, vol. 24, no. 2, pp. 177-185, 1996.

[9] T. K. Lee, "Semiprime rings with differential identities," Bulletin of the Institute of Mathematics. Academia Sinica, vol. 20, no. 1, pp. 27-38, 1992.

[10] I. N. Herstein, Topics in Ring Theory, The University of Chicago Press, Chicago, Ill, USA, 1969.

[11] Ö. Gölbaşı and E. Koç, "Generalized derivations on Lie ideals in prime rings," Turkish Journal of Mathematics, vol. 35, no. 1, pp. 23-28, 2011.

[12] T.-K. Lee, "Derivations with Engel conditions on polynomials," Algebra Colloquium, vol. 5, no. 1, pp. 13-24, 1998.

[13] T.-L. Wong, "Derivations with power-central values on multilinear polynomials," Algebra Colloquium, vol. 3, no. 4, pp. 369378, 1996.

[14] C.-L. Chuang, "GPIs having coefficients in Utumi quotient rings," Proceedings of the American Mathematical Society, vol. 103, no. 3, pp. 723-728, 1988.

[15] W. S. Martindale, III, "Prime rings satisfying a generalized polynomial identity," Journal of Algebra, vol. 12, pp. 576-584, 1969.

[16] V. K. Kharchenko, "Differential identities of prime rings," Algebra and Logic, vol. 17, pp. 155-168, 1978.

[17] K. I. Beidar, W. S. Martindale, III, and A. V. Mikhalev, Rings with Generalized Identities, vol. 196 of Monographs and Textbooks in Pure and Applied Mathematics, Marcel Dekker, New York, NY, USA, 1996.

[18] C. L. Chuang, "Hypercentral derivations," Journal of Algebra, vol. 166, no. 1, pp. 39-71, 1994.

[19] C.-T. Yen, "Note on theorems on Herstein," Bulletin of the Institute of Mathematics. Academia Sinica, vol. 17, no. 3, pp. 205209, 1989. 


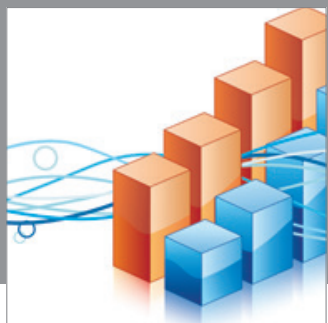

Advances in

Operations Research

mansans

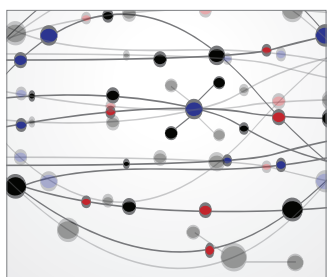

The Scientific World Journal
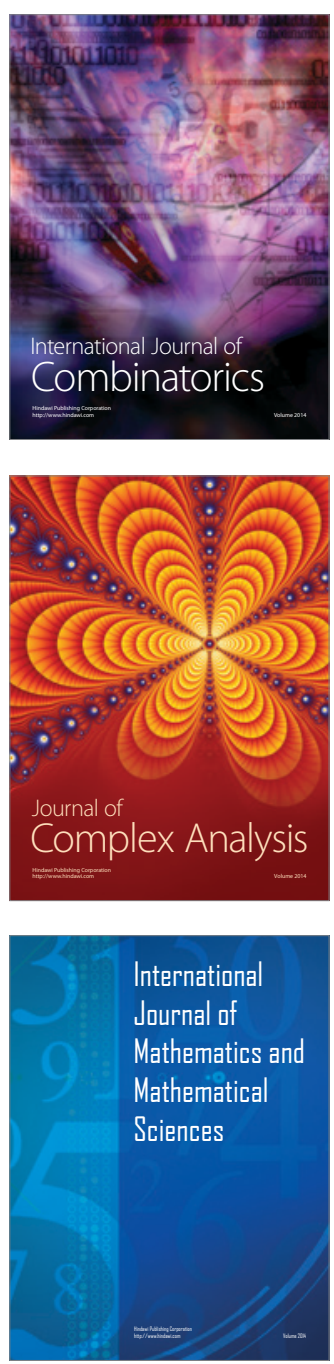
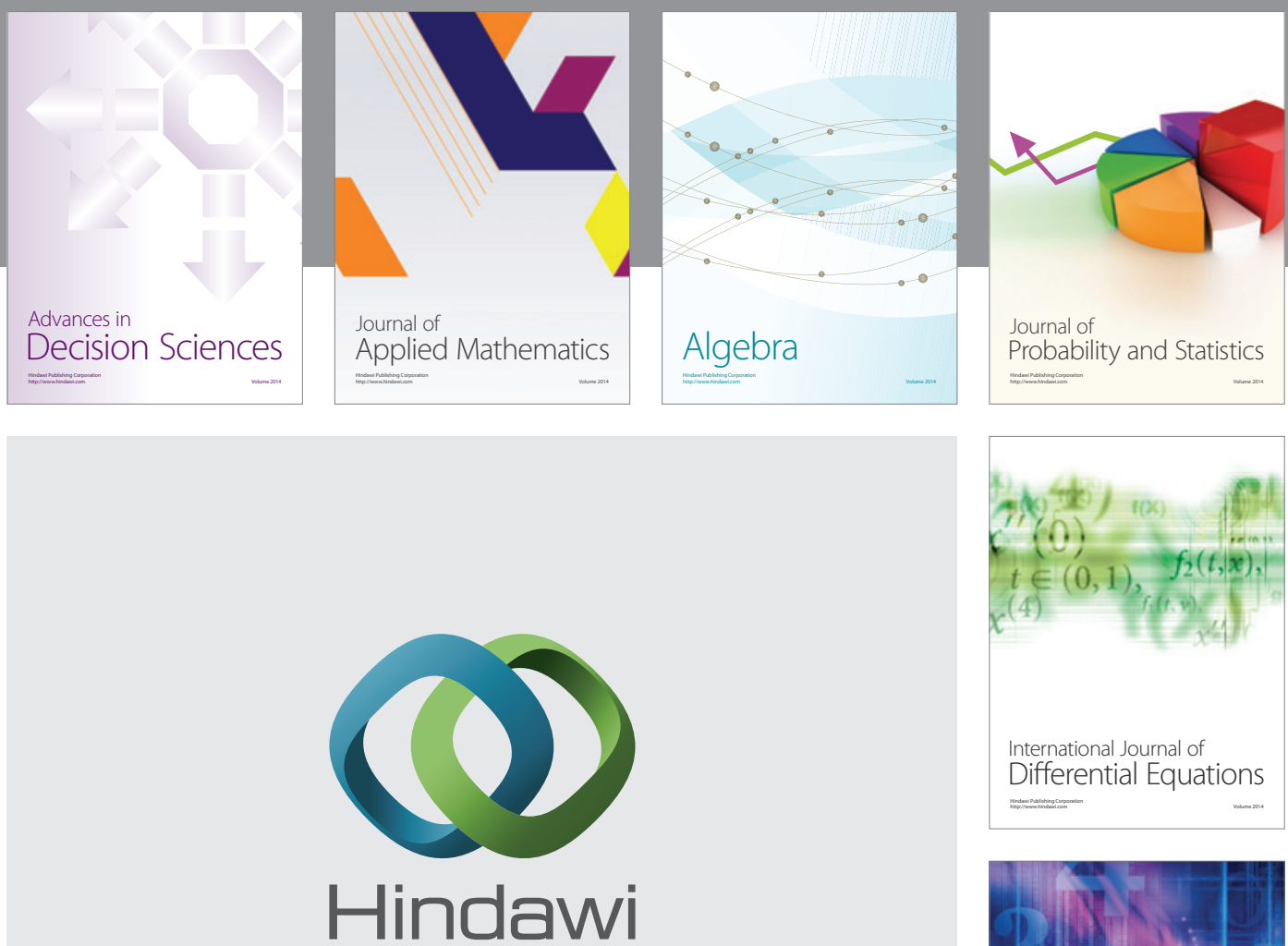

Submit your manuscripts at http://www.hindawi.com
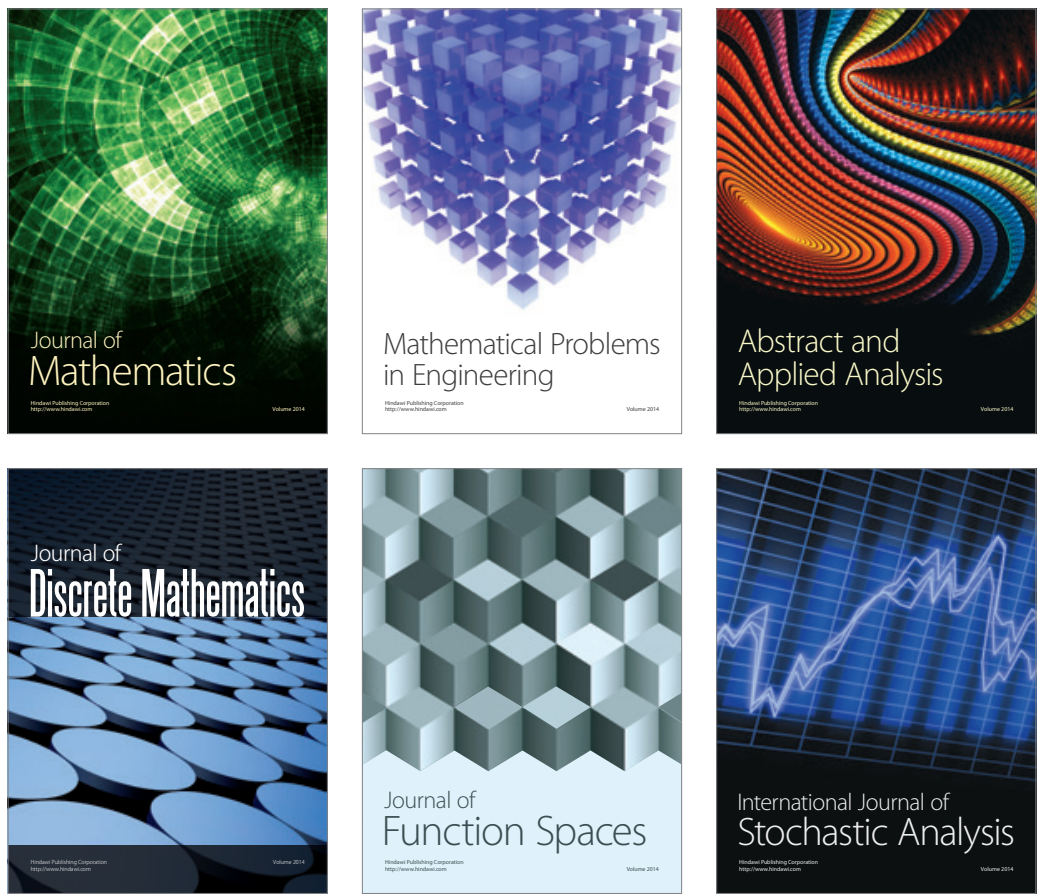

Journal of

Function Spaces

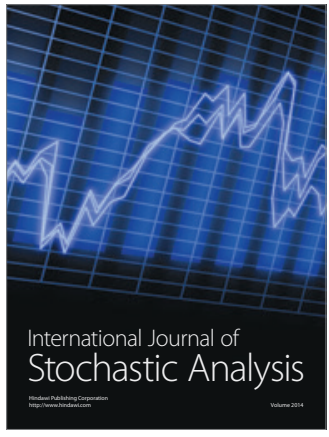

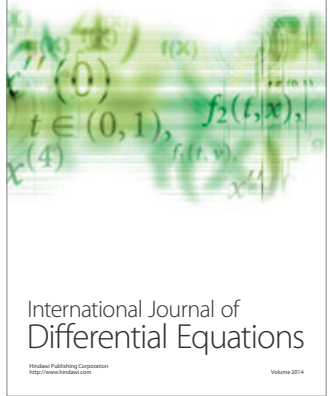
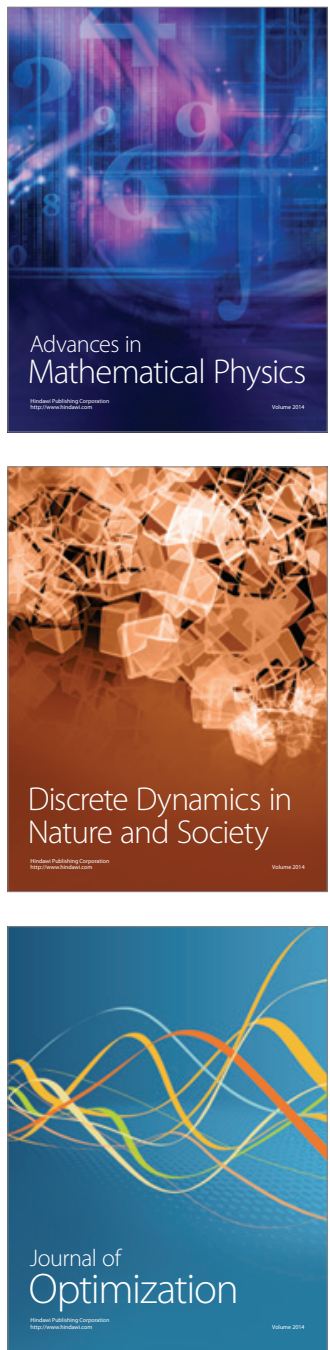